\title{
MOVIMIENTO DE ECONOMÍA SOCIAL Y SOLIDARIA DE ECUADOR. CIRCUITOS ECONÓMICOS SOLIDARIOS INTERCULTURALES
}

\author{
Jhonny Jiménez ${ }^{1}$
}

\section{Resumen/Abstract}

El presente artículo hace referencia a la construcción de los Circuitos Económicos Solidarios Interculturales (CESI), como una herramienta metodológica política para el fomento de sistemas económicos solidarios que promuevan la reproducción ampliada de la vida, en búsqueda del sumak ally kawsay (Buen Vivir). Los CESI parten del reconocimiento de la existencia de prácticas económicas ancestrales basadas en la reciprocidad y la redistribución, las cuales están presentes en las diferentes culturas, particularmente en los pueblos andinos. La propuesta de los CESI, al contrario de las cadenas productivas, se construyen en los territorios para satisfacer las necesidades fundamentales de las familias, impulsando prácticas productivas que promuevan el cuidado de la naturaleza, el consumo y la intermediación solidaria. La estrategia de los circuitos es la articulación de diversas prácticas solidarias a través del estímulo de su relación con el mercado y el trabajo en red.

Palabras clave: Circuitos, Economía Solidaria, territorio, consumo solidario, sociedades de mercado, interculturalidad, Buen Vivir (sumak ally kawsay).

1 Ecuatoriano. Universidad Central del Ecuador. E-mail: jhonnyjimenez@gmail.com 


\section{SOCIAL MOVEMENT OF ECUADOR AND SOLIDARITY ECONOMY. INTERCULTURAL CIRCUITS OF SOLI- DARITY ECONOMY}

This article refers to the construction of Economic Solidarity Intercultural Circuits (CESI) as a methodological political tool to promote economic systems that advance solidarity expanded reproduction of life, in search of Good Living (sumak kawsay ally). The CESI based on the recognition of the existence of ancient economic practices based on reciprocity and redistribution, which are present in different cultures, particularly in the Andean peoples. The proposal of the CESI, contrary to the productive chains, are built in the territories to meet the basic needs of families, promoting productive practices that foster care for nature, consumption and solidarity intermediation. The strategy of the circuits is the articulation of various solidarity practices through encouragement of their relationship with the market and networking.

Keywords: Circuits, Solidarity Economy, territory, solidarity consumption, market societies, multiculturalism, Good Living 
La Constitución ecuatoriana en su artículo 288 establece que el sistema económico ecuatoriano es social y solidario, colocando a la economía solidaria al mismo nivel que la economía pública y privada. El fin es la promoción de un nuevo modelo de desarrollo que la misma constitución denomina Sumak Kawsay o Buen Vivir.

Este reconocimiento constitucional es el resultado del esfuerzo de las organizaciones sociales que promueven ancestralmente prácticas económicas solidarias y que por otro lado se han movilizado políticamente para que el estado las reconozca como una nueva propuesta de desarrollo económico, social y político. Para la construcción de esta nueva sociedad basada en la solidaridad y la convivencia entre los seres humanos y el planeta, se plantea que la fuerza de la comunidad a través de su organización genera transformaciones políticas que buscan formas de relaciones más humanas.

Se ha planteado como abordaje conceptual, metodológico y estratégico la construcción de los Circuitos Económicos Solidarios Interculturales (CESI) como mecanismo para la generación de una nueva forma de desarrollo de los territorios que, a diferencia de otras formas de pensamiento económico como el desarrollismo, no se centra en el ámbito económico, sino que interviene en varias dimensiones: ecológicas, sociales, culturales, espirituales y políticas, basadas en los principios de solidaridad, reciprocidad, complementariedad y el cuidado de la vida, buscando satisfacer las necesidades humanas para asegurar la reproducción de la vida.

El presenteartículoesun primer intento para contribuir a la conceptualización de los Circuitos Solidarios Interculturales que el Movimiento de Economía Social y Solidaria del Ecuador (MESSE), desde el 2010, ha venido construyendo como un aporte al abordaje teórico de la economía solidaria, la que nace a partir de las experiencias que diversas organizaciones y comunidades vienen impulsando en el Ecuador, y que manifiesta los desafíos que se presentan a las organizaciones a la hora de seguir fomentado circuitos que generen procesos de transformación social y política. 


\section{El concepto de economía solidaria}

El concepto de economía solidaria está en constante construcción; aunque su terminología aparece ya en la década de los 80 (Razeto., 2009). Se ha desarrollado en América Latina, nutrida a partir de las diversas formas y prácticas que surgen de procesos sociales y culturales de subsistencia, así como también de prácticas ancestrales de resistencia a los modelos occidentales capitalistas, sobre todo relacionados con las experiencias de economía comunitaria, que en el caso del Ecuador tienen una trayectoria significativa. La economía social se fundamenta también en aquellas experiencias que nacieron como propuestas alternativas para la transformación política de los modelos autogestionados de libre mercado que se caracterizan por ser individualistas, racionalistas y utilitaristas.

El concepto de solidaridad forma parte del surgimiento de la sociedad democrática liberal, como parte de los procesos de reivindicación social, particularmente de los derechos instaurados a partir de la segunda guerra mundial y del surgimiento del Estado de Bienestar; donde aparecen, por un lado, una solidaridad filantrópica y por otro una solidaridad que Laville denomina democrática, la misma que fue reprimida permanentemente en de América del Sur en la década de los sesenta y ochenta. (Laville, 2006). Ahora bien, las prácticas solidarias estaban presente desde tiempos ancestrales en las comunidades y pueblos del sur y eran parte de la cotidianidad de las comunidades indígenas, enmarcadas en los principios de la reciprocidad y la complementariedad,

La economía solidaria está basada en algunos principios fundamentales: el trabajo autogestionado, la distribución-redistribución de los recursos, el cuidado del medio ambiente, la reciprocidad, la democracia, entre otros. Estos principios dan una orientación, un horizonte para que las prácticas solidarias se conviertan en una propuesta emblemática y paradigmática para la construcción de nuevas alternativas económicas, políticas y culturales. Es una propuesta paradigmática frente al modelo desarrollista occidental, ya que promulga un proyecto comunitario de convivencia entre las personas y la naturaleza, planteando nuevas relaciones sociales que se fundamentan en el vínculo no solamente entre seres humanos sino de ellos con la naturaleza. Por ello coloca en el centro de la actividad económica al trabajo autogestionado (Coraggio, 2011), la satisfacción de las necesidades y la no acumulación de los recursos, con un contenido ético-valórico, constituyéndose en una propuesta emblemática de una nueva sociedad. 
La economía solidaria recupera el sentido de la economía entendida como "oikonomia", que viene de dos palabras griegas el oiko que quiere decir "la casael planeta" y nomia que quiere decir "administración", es decir la administración y cuidado de nuestra casa en donde los seres humanos estamos incluidos como un componente más del universo; en base a esto la economía solidaria busca la reproducción material e inmaterial, no solamente de los seres humanos, sino de todas las especies que habitan en planeta.

Por ello la economía solidaria se aparta del concepto convencional de economía impuesta por las doctrinas clásicas y neoclásicas, las cuales señalan a la economía como la ciencia de la escasez, en la que el individuo racional toma las decisiones para maximizar las utilidades, en la que el fin justifica los medios y la naturaleza es vista como un recurso a ser explotado, preceptos asumidos por los economistas positivistas, así como por los llamados formalistas En contraste, la economía solidaria tiene su origen en los conceptos substantivistas de la economía relacionada con el "oikos", en la cual los marcos sociales, históricos y culturales son parte de las decisiones económicas (Molina, 2004).

Cabe recordar que la economía convencional que ha promovido el homo economicus racional utilitarista es reciente (Polanyi, 1944). Históricamente, la economía es una ciencia que es parte de las relaciones sociales, culturales y políticas. Hay una serie de estudios antropológicos y sociológicos, por ejemplo, los realizados por Mauss (Mauss, 1979) donde se establecen los conceptos de reciprocidad, el significado del don y los procesos sociales de intercambio, que sostienen que los procesos económicos no están caracterizados solamente por elementos utilitaristas, sino más bien juegan otros elementos como el prestigio y la construcción de convivencia. En ese mismo sentido, Godelier en los estudios con el pueblo baruya plantea que el intercambio de los objetos corrientes y sagrados (Godelier, 1998) está caracterizado por el carácter institucional total en los procesos de circulación (Molina, 2004) determinantes en las configuraciones sociales de las economías. Estos procesos que abarcan desde los principios de integración hasta patrones institucionales, planteados por Polanyi, son configuraciones sociales históricas donde se establecen normas, estructuras y reglas desarrolladas por las colectividades. El modo en el que llegaron a construirse da razón de la dimensión política de éstas prácticas (Wanderley, 2015), la que incluye dimensiones culturales y espirituales.

Los principios de integración y patrones institucionales están determinados por cuatro principios que Polanyi describe y que son recogidos por Wanderley, estos principios obedecen a construcciones históricas que se elaboran tanto en 
procesos de subsistencia como de intercambio. Entre ellos están el principio de redistribución que plantea la necesidad de una centro (estado) para generar la redistribución, dando lugar a mecanismos que a través de una autoridad política fomente la cohesión social; el principio del intercambio con competencia, dentro de una institución principal que es el mercado en el cual se desarrollan las acciones de compra y venta, y en el que se establece que su motivación principal es la generación de excedente que puede asumir tres modalidades: con fines de lucro, con fines de lucro limitados y sin fines de lucro; la reciprocidad que se fomenta entre pares y redes y que se desarrollan en procesos simétricos y horizontales; el de subsistencia para la sostenibilidad del núcleo familiar y de su entorno bajo la lógica de la reproducción de la vida (Wanderley, 2015). Estos principios se encuentran interrelacionados y obedecen a los patrones socio-culturales en cada uno de los entornos.

Estos elementos se encuentran vigentes en las prácticas solidarias que se realizan en diversos territorios, particularmente en las comunidades andinas y otras regiones. Por un lado, los principios de la reciprocidad, complementariedad y vincularidad son elementos constitutivos y están relacionados con las economías de subsistencia. Además, tienen un fuerte contenido contra-hegemónico, de energía transformadora de las sociedades modernas capitalistas. Para Razeto la economía debe incorporar la solidaridad y el trabajo como elemento esencial de las prácticas económicas. (Razeto, 2009). De este modo, en los procesos de producción, comercialización y consumo, la solidaridad se convierte en una fuerza transformadora como conjugación de los factores de la producción. Particularmente está presente el llamado Factor C (cooperación, cuidado, común) que se transforma en una categoría económica que organiza y conjuga a los demás factores productivos (Razeto, 1988; Guridi, 2014).

Otra de las corrientes importantes para la definición del concepto de economía solidaria viene del Foro Brasileño de Economía Solidaria, donde se pone énfasis en los procesos de articulación y construcción de redes con el fin de desconectarse de los sistemas de mercado; en ese camino Mance plantea a las redes de colaboración solidaria como mecanismos de articulación económica, social y política (Mance, 2008).

En el caso ecuatoriano las definiciones sobre economía solidaria se han configurado a través de la constitución cuyo Art. 283 plantea que el sistema económico es social y solidario, coloca al ser humano en el centro de la actividad económica, satisfaciendo sus necesidades materiales e inmateriales. Establece que el sistema económico está compuesto por tres sectores: público, privado, y popular- 
solidario, colocando al mismo nivel las tres economías. Esto es fundamental en la perspectiva de institucionalizar la economía solidaria en el Ecuador.

Hay que resaltar la importancia de la economía feminista, la que tiene como elemento central el cuidado de la vida y el cuidado doméstico, pero además los otros cuidados, como el de los enfermos, los adultos mayores y habría que añadirle el cuidado del planeta. Un elemento principal que plantea la economía feminista es la eliminación de la división sexual del trabajo productivo-hombres y reproductivomujeres. Para generar procesos de igualdad es necesario que los diversos géneros se dediquen al cuidado de la vida de manera integral, cuestionando también la división del trabajo público y privado. Sin embargo, la economía feminista y las asimetrías que se dan por los roles de género son temas ausentes en la economía solidaria, que desde América Latina enriquece su sustento teórico.

Por otro lado, es necesario resaltar los aportes de la economía ecológica que realiza un cuestionamiento a los modelos neoclásicos como la Ley de Say, en la que la oferta determina la demanda, el laisser faire, dejar hacer, dejar pasar y los modelos racionalistas, así como el flujo circular de la renta. Este modelo considera a dos actores en el proceso económico, las familias y las empresas en función de la utilidad y la maximización de las ganancias. La economía ecológica plantea la necesidad de incorporar en los flujos circulares de la renta y de bienes y servicios, los flujos energéticos que aseguren la sostenibilidad (Hauwermeiren, 1999) considerando a la naturaleza como un sujeto de derechos.

En el caso particular del Ecuador y de los países andinos, la economía solidaria también tiene sus orígenes en la economía comunitaria que promueve el Sumak Ally Kawsay o Con-Vivir Bien, que considera los principios de la complementariedad, la reciprocidad y vincularidad (entre las personas y la naturaleza), convertida en el eje de la acción humana, en contraposición a los modelos neoclásicos en los cuales la acción del individuo determina la acción económica. Incorpora también un modelo de producción comunitario definido como una "dinámica productiva y productora activada por el poder del conjunto complejo de reciprocidades entre los seres vivos que acuerdan en mantenimiento comunitario del Sumak Ally Kawsay" (Andrade, 2005: 187). En este sentido el nosotros genera procesos de socialización a través de la institucionalización de normas, prácticas y sentidos que se generan en torno a la familia y la comunidad.

No se pueden dejar de lado los aportes que realiza la cultura de la paz en donde la convivencia se convierte en el eje de las relaciones, planteando la necesidad de promover relaciones simétricas sean éstas de género, intergeneracionales, 
ambientales, étnicas y sociales, caracterizadas por los principios de la interculturalidad, la integración, la solidaridad, el respeto y la armonía. La cultura de paz no es un estado de tranquilidad, es principalmente la eliminación de todas las formas de violencia, para lo cual hay que generar procesos de construcción políticos, históricos y culturales.

En ese mismo entorno conceptual, la Ley de Economía Popular y Solidaria publicada en el 2011 en Ecuador entiende por Economía Popular y Solidaria a "la forma de organización económica, donde sus integrantes, individual o colectivamente, organizan y desarrollan procesos de producción, intercambio, comercialización, financiamiento y consumo de bienes y servicios, para satisfacer necesidades y generar ingresos, basadas en relaciones de solidaridad, cooperación y reciprocidad, privilegiando al trabajo y al ser humano como sujeto y fin de su actividad, orientada al buen vivir, en armonía con la naturaleza, por sobre la apropiación, el lucro y la acumulación de capital." Art. 1 (Asamblea, Nacional, 2011).

En el mismo sentido, el Movimiento de Economía Solidaria define a la economía solidaria como un concepto transformador de los modelos económicos gestionados por modelos de mercados autorregulados, pero además plantea como eje principal los procesos organizativos como energía transformadora, así como también incorpora elementos de las prácticas ancestrales que devienen de la economía comunitaria, estableciendo la necesidad de fomentar la cultura de paz. (MESSE, 2005)

Las prácticas solidarias son configuraciones económicas determinadas por principios, normas, reglas y formas organizativas que se generan en procesos sociales históricos, pero también geográfico-territoriales, las cuales tienen un contenido ético transformador y contra hegemónico para la construcción de una económica con ética. Por ello la economía solidaria se convierte en una propuesta paradigmática que conlleva nuevas configuraciones sociales, prácticas e instituciones que permiten promover una nueva sociedad en el centro la reproducción de la vida, en todos los sentidos. No es una propuesta utópica, sino prácticas reales que están resolviendo los problemas fundamentales, sean estos en la reproducción material e inmaterial de la fuerza de trabajo, pero también en problemas ecológicos, financieros, valóricos y éticos.

Las prácticas solidarias se desarrollan en los territorios y en ellos se han generado procesos de producción, comercialización y consumo donde se pueden encontrar nuevas formas de relaciones sociales conjugados con los factores de 
la producción en función del servicio de la vida. A partir de los territorios se construyen los circuitos económicos solidarios como una propuesta política, cultural y social, así como metodológica, para generar procesos de articulación de las prácticas solidarias a nivel local.

En ese sentido, el Movimiento de Economía Social y Solidaria, MESSE, viene trabajando desde los territorios en la construcción de los circuitos solidarios como una estratégica de fortalecimiento del sector de la economía solidaria en el Ecuador.

\section{El concepto de los Circuitos Económicos Solidarios (CESI)}

Como se había manifestado anteriormente, el mercado autorregulado determinado por la ley de Say, donde la oferta determina la demanda, laisser faire, laisser passer (dejar hacer, dejar pasar), se encuentra institucionalizado en las prácticas e instituciones, generando actitudes y comportamientos utilitaristas y globalizando un sentido común que para Helio Gallardo se define como "producir con eficiencia-consumir con opulencia" (Gallardo, 2001). Sin embargo, en el mundo y en particular en Latinoamérica, frente a las propuestas totalitaristas y autoritarias de mercado autorregulado, históricamente han existido formas comunitarias basadas en principios de reciprocidad y redistribución, que en términos históricos han permanecido más tiempo que las prácticas del mercado actual. (Polanyi, 1944) (Mauss, 1979) (Godelier, 1998) (Guerra, 2005).

Muchas de estas prácticas se encuentran presentes en las economías comunitarias $y$, en los últimos años, se ha generado una serie de luchas sociales que han tenido como resultado el fortalecimiento de una solidaridad democrática teniendo como elemento principal a las economías plurales, poniendo de manifiesto que en el mundo existe una diversidad de formas económicas que se han resistido a una sociedad de mercado.

En el caso de nuestra región, estas propuestas alternativas de economía aparecen como formas de resistencia comunitaria frente a los procesos de colonialismo, siendo también una alternativa de auto subsistencia y de transformación social, las mismas que están marcadas por instituciones y prácticas cotidianas que recuperan los principios de la reciprocidad, complementariedad y vincularidad, que han permitido soportar los embates de procesos económicos neoliberales. 
Estas prácticas solidarias vienen de la economía popular e informal, pero también de economías comunitarias que no han dejado de fomentar prácticas como el randa-randi, el presta manos, el trueque y el fio, las que dan cuenta que los principios de la reciprocidad, redistribución y complementariedad están presentes. Estas prácticas en los barrios urbano marginales de las ciudades se encuentran presentes en menor medida, principalmente por el sincretismo producto de la migración de población indígena y campesina a las urbes que trasladaron sus prácticas comunitarias al mundo urbano (Jiménez, 1996).

Por la implementación de un modelo totalitario estas prácticas solidarias se encuentran invisibilizadas y tampoco son reconocidas como un aporte a la economía del país, por ende, no se establecen políticas públicas que las potencien. Los modelos económicos que dominan, el de la libre oferta y demanda han establecido un entramado de políticas y leyes que favorecen la propuesta económica y política de acumulación, ganancia y poder, excluyendo todas las formas alternativas de economía. Sin embargo, estas formas alternativas de economía cobran fuerza frente a la crisis permanente del sistema neoliberal que no solo es económica, sino climática, financiera y de valores. Los movimientos sociales se han pronunciado para que se incorporen estas nuevas visiones y modelos de desarrollo que colocan en el centro a la reproducción de la vida.

En el Ecuador, la presión de los movimientos sociales hace que en el 2008 se incorpore a su constitución una nueva mirada de ver el modelo económico, señalando que es social y solidario. Esto es de vital importancia ya que denota la construcción de nuevas relaciones sociales de producción, las que deben estar enmarcadas en los principios de la solidaridad: reciprocidad, redistribución, complementariedad, la participación, y la democracia. El fomento de fuerzas productivas debería estar al servicio de la vida, considerando el factor trabajo y el asociativo como categorías centrales de la economía.

También implica una nueva institucionalidad, con normas, leyes y formas de organización, así como la generación de nuevas sensibilidades sociales, nuevos sentidos comunes al servicio de la vida. Estos serán el producto de formas de socialización, donde la educación y la comunicación jueguen roles importantes en la generación de actitudes, cosmovisiones y valores caracterizados por el principio de solidaridad.

Este no es una tarea fácil, frente a un mundo globalizado en donde priman los conceptos utilitaristas de maximización de la ganancia que produce una 
concentración de la riqueza cada vez mayor, donde el consumo superfluo y exacerbado es parte de la cotidianidad y donde las leyes están al servicio de la transnacionalización de la economía. En ese escenario adverso, la economía solidaria se presenta como una alternativa en crecimiento, cuyo problema no es solamente económico, sino que entra en la esfera política, cultural, social, por no decir espiritual.

La construcción de una economía con solidaridad parte desde experiencias concretas que se desarrollan en territorios donde se afincan éstas para satisfacer las necesidades fundamentales a través de satisfactores sinérgicos (Neff, 1999). Las propuestas alternativas económicas deberían nacer de procesos concretos, pensar primero en lo local, nacional y regional para luego pensar internacionalmente. Una forma de desconectarse del sistema económico de mercado es fortaleciendo las redes de colaboración solidaria en donde las diversas prácticas que trabajan en función de estos principios se asocien para producir-distribuir-consumir de manera alternativa.

Para Coraggio, citado por Wanderley, la manera de convertirse en un actor de incidencia social y política es avanzando a un segundo nivel de organización donde "la eficacia social y la sostenibilidad de las iniciativas económicas impulsadas, solo encontrarán en las redes de solidaridad sociales y políticas, condiciones para la formación como sujetos colectivos... constituidos en un sector orgánico" (Wanderley, 2015: 65). Para alcanzar el fomento de "otra economía" se debería "avanzar hacia el desarrollo de otras economías integradas en la sociedad sobre la base de relaciones de solidaridad, de justicia y de igualad, orientadas por el criterio común de la reproducción ampliada de la vida" (Wanderley, 2015: 65), elementos para fortalecer la acción social con capacidad de incidencia, permanencia y una propuesta política emancipadora.

En ese proceso, el Movimiento de Economía Social y Solidaria del Ecuador plantea el concepto de los circuitos económicos solidarios interculturales (CESI) como una propuesta conceptual-metodológicaestratégica para el fomento de un sistema económico solidario que fomente el Sumak Ally Kawsay desde los territorios, los cuales puedan disputar sentidos, pero también flujos de la economía real y monetaria para disminuir el poder hegemónico de una sociedad de mercado y la construcción de una sociedad con mercado. Al respecto, Armando Melo Lisboa, citado por Pablo Guerra, manifiesta: "El desafío de la economía de la solidaridad consiste entonces en superar esa sociedad de mercado, sin renegar de los mecanismos mercantiles: ello sólo será posible por medio de la construcción de circuitos de intercambio 
solidarios entre emprendimientos, de forma de ir configurando otro mercado" (Guerra, 2005: 47).

Las experiencias de economía solidaria tienen desafíos que enfrentar; uno de ello es que las políticas institucionales que vienen del estado no se encuentran al servicio de dicha economía, lo que no le permiten generar procesos políticos y económicos de mayor alcance.

La construcción de los CESI nace de la necesidad de articular las prácticas de economía solidaria por un lado y vivenciar los principios de ésta para que se fomenten las relaciones sociales basadas en los principios de la reciprocidad, distribución y cuidado del medio ambiente, además de la construcción de autonomía política, cultural y social que le permita des-imbricarse de la sociedad de mercado. En este sentido los CESI promueven la articulación de las prácticas y actores de la economía solidaria relacionados a los campos de producción sana, finanzas solidarias, el comercio justo, el consumo responsable, el post-consumo, el turismo comunitario y la salud ancestral, que se adscriben a los principios de la economía solidaria y que deciden articularse para generar procesos políticos y económicos en pos de construir una sociedad del Buen Vivir (con-vivir) Sumak Kawsay. (Messe, 2005)

Gonzalo Silva define a los CESI como "un conjunto integrado de unidades socio-económicas que se adscriben a criterios de la economía solidaria, las cuales pueden ser conformadas a distintos niveles de agregación y tienen vínculos complejos con el entramado social, cultural y político del territorio. Los CESI operan según procesos sistémicos de producción, transformación, servicios, comercialización, consumo y reciclaje y conforman por tanto el sector de la E.S. que al articularse pueden constituirse como un nuevo sujeto político local." (Silva, 2012: 48) En ese sentido los circuitos son flujos económicos de bienes y servicios para satisfacer las necesidades de las personas, por ende, son políticos, culturales y sociales, articulados en función de los principios de la solidaridad. Son formas de acumulación que no solo hacen referencia al capital sino a otras dimensiones como culturales, políticas y sociales, que permiten el desarrollo de capacidades individuales y colectivas. (Lopera \& Mora, 2009)

Un factor importante en la constitución de los circuitos es la vivencia de los principios de la economía solidaria que generan procesos de articulación para el fomento de la convivencia y la construcción de comunidad, que, desde 
la cultura andina, están bajo el paraguas del paradigma Abya Ayala ${ }^{2}$, en el que la base fundamental es la vincularidad entre las personas (nosotros) y la naturaleza. Por ende, los circuitos tienen mayor facilidad de expansión donde se puede vivenciar estos principios en la cotidianidad, por ejemplo, en las comunidades, barrios y las formas económicas alternativas.

Para el fomento de circuitos es necesario construir normas de convivencia que promuevan nuevas sociabilidades basadas en los principios de la economía solidaria, como los de complementariedad y reciprocidad, y que permitan, según Zabala, procesos de responsabilidad, funcionalidad, racionalidad (Zabala, 1997) para generar procesos efectivos que satisfagan necesidades fundamentales. Según Mance, la construcción de circuitos se basa en procesos de articulación a través de redes de colaboración solidaria, la cual implica una articulación entre diversas unidades que, a través de ciertas conexiones, intercambian elementos entre sí, con lo cual se fortalecen recíprocamente y se pueden multiplicar en nuevas unidades. A su vez, dichas unidades fortalecen todo el conjunto, permitiéndoles expandirse en nuevas unidades o mantenerse en equilibrio sustentable. Cada nódulo de la red representa una unidad y cada hilo un canal por donde esas unidades se articulan a través de diversos flujos. (Mance, 2008: 38) La construcción de las redes parte del fomento y promoción el consumo solidario, la generación de procesos de producción y mercados solidarios que permitan satisfacer las necesidades, partiendo de que somos prosumidores, es decir, a la vez somos productores de bienes y servicios y consumidores.

En suma, los principios que se fomentan desde los CESI y que están relacionados con los de la economía solidaria son:

- La reciprocidad-complementariedad y la redistribución.

- La vincularidad, entre las personas y la naturaleza.

- La asociatividad, la organización, el trabajo colectivo comunitario.

- La construcción de autonomía.

Reciprocidad, complementariedad, redistribución, vincularidad y organización

Un principio importante para los CESI es la reciprocidad entendida como el acto de dar y recibir. Desde la cosmovisión andina, se entrega productos y

2 El término Abya Yala fue colocado por los pueblos Kunas de Panamá y Colombia para nombrar al territorio americano, 
servicios con el fin de hacer el bien a los demás y a la naturaleza, no se da para recibir, sino que se lo hace por el hecho de entregar lo que se ha recibido de la Pachamama (madre tierra-universo); según la percepción circular del cosmos, el hecho de "dar, algún día recibiré".

La reciprocidad es un acto de sujetos sociales que se miran, actúan y se tratan como tales, es decir, existen relaciones simétricas y horizontales, se entrega como un acto que fomenta la comunidad. La reciprocidad no es caridad en las que hay relaciones de poder desiguales: "yo que tengo más entrego al otro que no tiene, el pobrecito que necesita". En la reciprocidad me asumo como un ser necesitado de solidaridad, el dar me forma como ser humano, pero miro al otro también como un sujeto, esa relación humana se convierte en un acto político de construcción de comunidad: necesito de los otros para fomentar la reciprocidad que me constituye como persona, en palabras de Helio Gallardo, "quererse con los otros y para los otros".

La reciprocidad fomenta los circuitos solidarios ya que fortalece las articulaciones que se generan en los actos de intercambio, produciéndose encuentros horizontales y simétricos. La reciprocidad se produce en todo momento y no solo donde se intercambian productos y servicios, en estos están inmersos actos espirituales, corporales, emocionales (un abrazo, una sonrisa) que se convierten también en productos de la reciprocidad.

Dentro del espacio del mercado socialmente organizado, se convierte a la reciprocidad en un acto político de transformación social. En palabras de Polanyi:

"La reciprocidad denota movimientos entre puntos correlacionados de grupos simétricos; redistribución indica movimientos de apropiación hacia un centro y consecutivamente fuera de él; y el intercambio se refiere a un intercambio entre manos que sucede dentro de un sistema de mercado. La reciprocidad requiere de un contexto de grupos organizados de forma simétrica; la redistribución depende de la presencia de alguna medida de centralidad en el grupo y el intercambio, si es que va a producir alguna forma de integración, requiere un sistema de precios fijados en el mercado". (Polanyi, 1958.) $)^{3}$

Soy recíproco con la naturaleza al comprar productos agroecológicos,

3 Enunciado por Verónica Andino, Cuaderno de Economia Solidaria, para la Incidencia y el diálogo de Saberes: 29. 
cuando decido pagar un precio justo, cuando intercambio bienes y servicios, cuando hago trueque, etc.; es decir, cuando se incorpora la reciprocidad como parte de mis acciones conscientes puedo generar procesos de transformación social.

Para Pereira (Pereira, 2012), la reciprocidad va de la mano de la redistribución como elemento central que permite a las personas satisfacer necesidades humanas fundamentales, la redistribución genera procesos de igualdad y equidad. La igualdad vista como un principio que permite a las personas vivir dignamente, satisfaciendo sus necesidades fundamentales, pero también la equidad en el sentido de darle a las personas y grupos humanos lo que necesitan.

La acumulación tiene que ser distribuida en la comunidad (para que no se genere concentración de poder y recursos) para permitir que todos vivan en mejores condiciones; por ejemplo, en las comunidades indígenas la producción que se tiene es repartida entre ellas: "Si hay un excedente en la producción, éste es redistribuido en el contexto de las necesidades materiales, los festivales y los rituales de la comunidad." (Delgado, 2015: s/p) Además, la reciprocidad va de la mano de la complementariedad, el sentido de que, si una persona o comunidad no dispone de algún producto, el resto de la comunidad se lo otorga. Se relaciona también con los procesos de intercambio entre pisos ecológicos, como el caso del Trueque en Pimapiro ${ }^{4}$ donde hay un proceso de intercambios entre la parte alta y baja del valle del Chota y de esta forma se satisfacen las necesidades fundamentales.

Al referirnos a la redistribución no solamente se piensa en el dinero, como generalmente se lo asocia en el ámbito económico, sino que también hace mención a saberes, conocimientos y productos. Es uno de los principios de la política pública para el fomento de los derechos, particularmente de los económicos, sociales y culturales, partiendo del principio que todos los bienes son de todos, es decir, comunitarios.

La Vincularidad debe ser entendida como la clave para el fomento de la vida, no solo con las personas, sino con los seres que forman parte de la naturaleza: plantas, animales, piedras, el cosmos, el universo.

La economía capitalista tiene como eje central y prioritario la ganancia y la rentabilidad, la cual mueve todas las intenciones y acciones; hasta parece

4 Comunidad de la sierra norte de Ecuador. 
ilógico que emprendamos una actividad si no existe ganancia o rentabilidad. Esta lógica fomenta la relación medio -fin, utilizamos todos los medios para llegar al fin deseado sin entender que durante ese camino se deterioran las relaciones familiares, la situación de los trabajadores, el medio ambiente y hasta la situación personal.

Si bien la economía solidaria no descarta la rentabilidad y la ganancia, sí coloca al ser humano y sus relaciones en el centro de su accionar, partiendo primero por satisfacer las necesidades fundamentales que le permitan una plena realización, teniendo en cuenta también las relaciones entre todos los participantes. En la economía solidaria no está excluida la vida espiritual; de hecho, en muchas de las prácticas se incorporan ceremonias que fomentan nuevas relaciones con Dios, con la naturaleza y el cosmos. Se fomentan el descanso y la risa y se busca el aprendizaje, potenciando las capacidades de cada cual. No son ajenos el ánimo, el modo de resolver los conflictos, la autoestima de cada cual ni las relaciones familiares. Las prácticas de economía solidaria se llevan a cabo de modo horizontal, respetando el disenso y se resuelven las diferencias a través del diálogo, sin violencia.

Uno de los elementos centrales de las prácticas solidarias es la organización: mientras más fuerte, más alto es el nivel solidario; la organización es el elemento principal que dinamiza los procesos de la economía solidaria. En el Ecuador encontramos una infinidad de prácticas solidarias que se han construido a partir de procesos organizativos - comunitarios como la minga y la realización de actividades en conjunto que han permitido resistir los embates de los modelos de dominación.

Generalmente, cuando se emprende una empresa lo que primero pensamos es el dinero que se necesita, en términos económicos el capital; en economía, al igual que el trabajo y la naturaleza, se les denomina factores productivos, que son la energía o la fuerza que permite que los bienes y servicios puedan producirse. Si no se tiene el dinero- capital difícilmente se puede emprenden una actividad económica, pero además con el dinero se obtienen otros factores como el trabajo (pagando un salario), comprar maquinaria - tecnología - y tener materias primas. En la economía capitalista el capital es considerado como uno de los principales factores productivos que tiene la empresa. En las prácticas de economía solidaria lo importante no es el capital sino la organización, mientras más organización existe más fuerte es, podríamos decir que sin este elemento no existe economía solidaria, aunque sean familiares, comunitarias o barriales. Es la organización la que permite pensar, organizar 
las habilidades, destrezas, administrar el dinero que se dispone para producir y consumir.

Existen muchos proyectos que financian iniciativas para emprender actividades económicas que tienen como objetivo salir de la pobreza, se busca personas, familias o grupos organizados para capacitarlas en emprendimientos, se les dota de pequeños capitales para montar sus negocios, pero muchas de estas experiencias han fracasado al no tener un grupo humano fortalecido, con lazos de confianza y unión, con la misma fuerza que nacen se derrumban. Al emprender alguna práctica solidaria la organización se convierte en el factor fundamental con el cual se debe empezar.

Razeto manifiesta que mientas más organización existe y de mejor la calidad, los productos son de mayor calidad, y pasa lo mismo con los otros factores, si los trabajadores están bien capacitados o la tecnología es buena, se obtienen mejores productos; lo mismo sucede con el Factor C, mientras se mejore la organización, ésta es más unida, se tienen normas de convivencia, procedimientos que se cumplen, se resuelven los problemas de manera positiva, existe confianza y unión, los intereses son grupales pero también se reconocen los individuales, el trabajo es reconocido, y entonces la calidad de los bienes y servicios que se producirán será de mejor calidad. Las decisiones se deben tomar de consenso y participativamente, aunque esto implique más tiempo.

Hay que resaltar que en el Ecuador existe una historia y una cultura de reciprocidad y solidaridad, que está unida al trabajo desarrollado por las organizaciones de la sociedad civil; existe una extensa y rica variedad de procesos organizativos que permiten que el Factor $\mathrm{C}$ sea más fácil de obtener; tenemos una cultura arraigada de trabajo comunitario que aprovechar y potenciar.

Un desafío para los CESI es la construcción de la autonomía como elemento para ser menos vulnerables, y es entendida como la capacidad para la toma de decisiones sin depender de actores o factores externos. Gandhi planteó en su programa constructivo, Poorna Swarja (pleno autogobierno) el fomento de la autonomía plena que permita a las comunidades y pueblos llegar a la independencia completa. De hecho, planteó el trabajo con el khadi como mecanismo para romper las relaciones de explotación y buscar la autonomía de los poblados en la India. "El uso del khadi implica el inicio de la libertad

5 El khadi es un mecanismo manual que permite a las comunidades de la india producir sus propias telas. 
económica y la igualdad de todos en el país." (Ameglio, 2002: 308). "Ello significa una mentalidad swadeshi (autosuficiencia), una determinación para encontrar todo los necesario para vivir en la India." (Ameglio, 2002: 309)

\section{La construcción de los circuitos económicos solidarios en los territorios}

El Movimiento de Economía Solidaria del Ecuador, desde el 2001 decidió apostar por la construcción de los CESI en los territorios que le permitan fortalecer procesos económicos para satisfacer las necesidades y procesos socio políticos para la construcción de sistemas económicos solidarios.

La experiencia que ha desarrollado el MESSE se basa en experiencias que vienen implementándose en los territorios donde actúa el Movimiento, considerando que éste es el lugar privilegiado para el fomento de los circuitos, particularmente en las zonas andinas y otras regiones donde algunas prácticas ancestrales Del Don se encuentran presentes como la reciprocidad, complementariedad y vincularidad. En la sistematización de los casos de los CESI por parte del MESSE se "evidenció la unidad intrínseca que existe en el mundo andino de los diversos ámbitos.... Todos son componentes que no se encuentran separados o disgregados en la práctica concreta o cotidiana; por el contrario, están unidos formado un todo, que es parte de la cosmovisión andina." (Yaselga \& Jara, 2012: 25)

Hablar de territorio no solamente se refiere a la tierra donde se habita, el territorio va más allá, es el lugar donde se conjugan una serie de elementos que permiten a las personas y a los grupos que habitan en ellos satisfacer sus necesidades, cumplir sus aspiraciones y fomentar la identidad. Por ende, el territorio comprende la tierra, el agua, la biodiversidad, los saberes, las costumbres, cosmovisiones, imaginarios, visiones, relaciones, sueños y esperanzas.

El territorio es determinante para comprender lo que somos como personas y grupos, en él se fomenta la identidad, el sentido de pertenencia. En el territorio se puede visualizar con certeza a las personas, los actores y las relaciones. Además, cuáles son las instancias de gobierno y quiénes son los que están al cargo de ellas, así como los entes con los que tenemos que interlocutar y acceder. Es allí en donde se pueden establecer alianzas, conocer las relaciones de poder o de confianza para establecer aliados e incidir políticamente para que las acciones cumplan con los objetivos planteados. 
Por ende, el territorio es el lugar privilegiado para el fomento de la participación, en donde los actores asumen roles activamente y determinan el tipo de territorio para vivir. Tiene varias dimensiones: físico (frontera), económico, cultural, político y social. En este sentido, el territorio es decisivo para fomentar un tipo de desarrollo, por ejemplo, se puede fomentar una economía pensada en la agroexportación o la explotación de la naturaleza. Pero también se podría impulsar otra economía que fomente, como dice la Constitución ecuatoriana, el Sumak Kawsay (Buen Con-Vivir).

Para (Andino, 2013) "Los CESI surgen en un territorio específico, su desarrollo no está limitado por un espacio geográfico local sino por la coincidencia con un proyecto de convivencia basado en el Sumak Kawsay. Por otro lado, los CESI no se limitan a una noción de especialidad pues en ellos suceden flujos, movimientos que valoran y recrean una dimensión temporal que, desde el paradigma del Sumak Kawsay, redefine los tiempos para el consumo, para las relaciones, para las conversas, para la ritualidad y la fiesta, para la construcción de lo político, etc. y que, por lo tanto, no están supeditados a la noción de tiempo-eficiencia ni de tiempo lineal omnipresentes en el pensamiento occidental."

Gracias al proceso implementado por el MESSE se puede establecer que los diferentes circuitos están determinados para la satisfacción de necesidades fundamentales de auto subsistencia. Las experiencias están presentes en función de valorizar la economía de trabajo, los conocimientos en los procesos de producción, gestión y comercialización, la generación de ingresos monetarios y no monetarios, además de la construcción de identidades en función de los principios de la solidaridad.

Se denotó que en las diversas prácticas de economía solidaria existían procesos de articulación solidarios débiles, es decir, que una familia que produce de manera agroecológica y vende sus productos en la feria de economía solidaria, termina comprando en el supermercado o en la tienda de barrio. De la misma manera, muchos productores compraban sus insumos en el mercado común capitalista donde se fomenta la explotación de los trabajadores y el daño del medio ambiente, pero además se denota que la mayoría de los inmensos recursos que se producen salen fuera de los territorios generando más procesos de empobrecimiento.

La falta de articulación y la salida de los recursos se deben, entre otros elementos, a que no se disponen de espacios donde se fomente la articulación, 
como es el caso de los mercados solidarios y, el trabajo en red que permitan asumirse como consumidores solidarios y profundizar los principios de la economía solidaria, tanto como consumidores y productores. Pero también se encuentran formas de articulación más fuertes donde existe un mayor control del proceso económico y donde los procesos de articulación y construcción de alianzas y redes son más fuertes, conllevando a que la construcción de autonomía tenga mayor sostenibilidad.

Existe una diversidad de prácticas solidarias que vienen de sectores de primer, segundo y tercer grado, por lo que los niveles de articulación son amplios y diversas, permitiendo mejorar los niveles de asociatividad, la construcción de alianzas, redes y niveles de incidencia económica y política. El manejo de los circuitos está determinado, según Silva (Silva, 2012), por el grado de activación del proceso económico en referencia al manejo y control del proceso económico, a la existencia de conexiones entre redes de aliados del sector -fundamentalmente a los que genera procesos económicos, sociales, culturales y políticos, que parten de que la alianza y construcción de redes no solo son económicos-, a la capacidad de gestión del circuito y sus partes, y al alcance y visibilización del sector solidario y de su identidad.

Las experiencias encontradas están asentadas en valores culturales, por ende, la interculturalidad se convierte en un eje de trabajo en función de las relaciones que se dan dentro de los actores, como por ejemplo las comunidades Shuar donde han mantenido valores culturales ancestrales y donde la reciprocidad, distribución, la complementariedad y la vincularidad se encuentran en la cotidianidad de la vida comunitaria, permitiendo vivir en ambientes armónicos entre las personas y la naturaleza.

Al referirse a la identidad de los circuitos, encontramos casos que construyen una identidad de economía solidaria, también hay casos donde la identidad histórica cultural es la que domina los procesos económicos y de relaciones sociales, otros en cambio tienen una identidad más urbana con un sentido político transformador donde el compromiso y la conciencia de las personas es un eje del trabajo; y finalmente, existe un componente de las entidades de apoyo como las fundaciones y las ONG's que juegan un papel de sostén, que es fundamental, pero que es vulnerable frente a los apoyos financieros que vienen del desarrollo. 

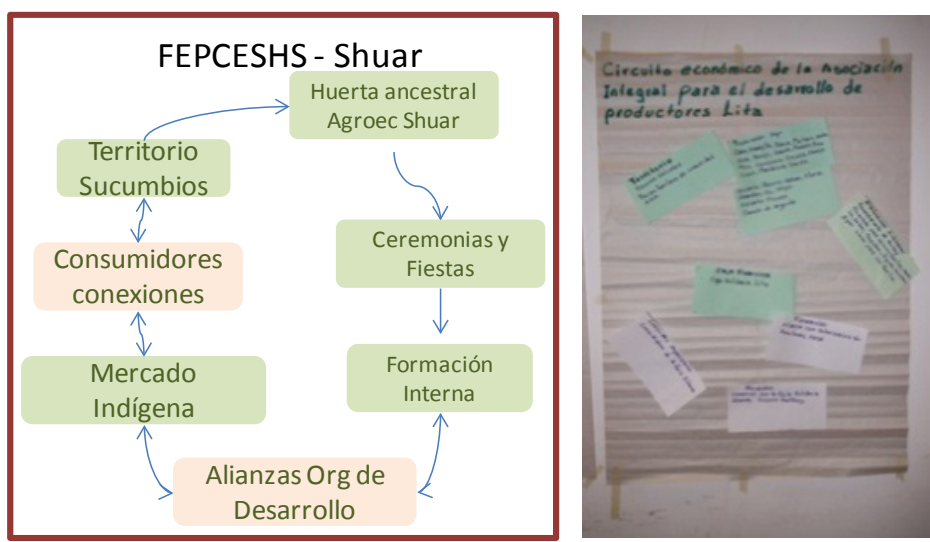

Fuente: Sistematización Proyecto Circuitos Solidarios Interculturales

Las prácticas de economía solidaria están presentes en todos los procesos de intervención del proceso económico, sean estos en los ámbitos de la producción, comercialización, consumo y pos-consumo, pero además articulan procesos como el turismo comunitario, las finanzas solidarias y la comunicación alternativa. Experiencias que dan cuenta del fomento de identidad y valor agregado, como el caso del Salinerito y Aprocuy, experiencias que van logrando mayores niveles de sostenibilidad, sean a nivel social, ambiental o financiero. El fortalecimiento del circuito se establece en el nivel de control- autonomía de los flujos monetarios y financieros, pero además en el establecimiento de normas y prácticas que nazcan de la dinámica de los procesos de los CESI. 

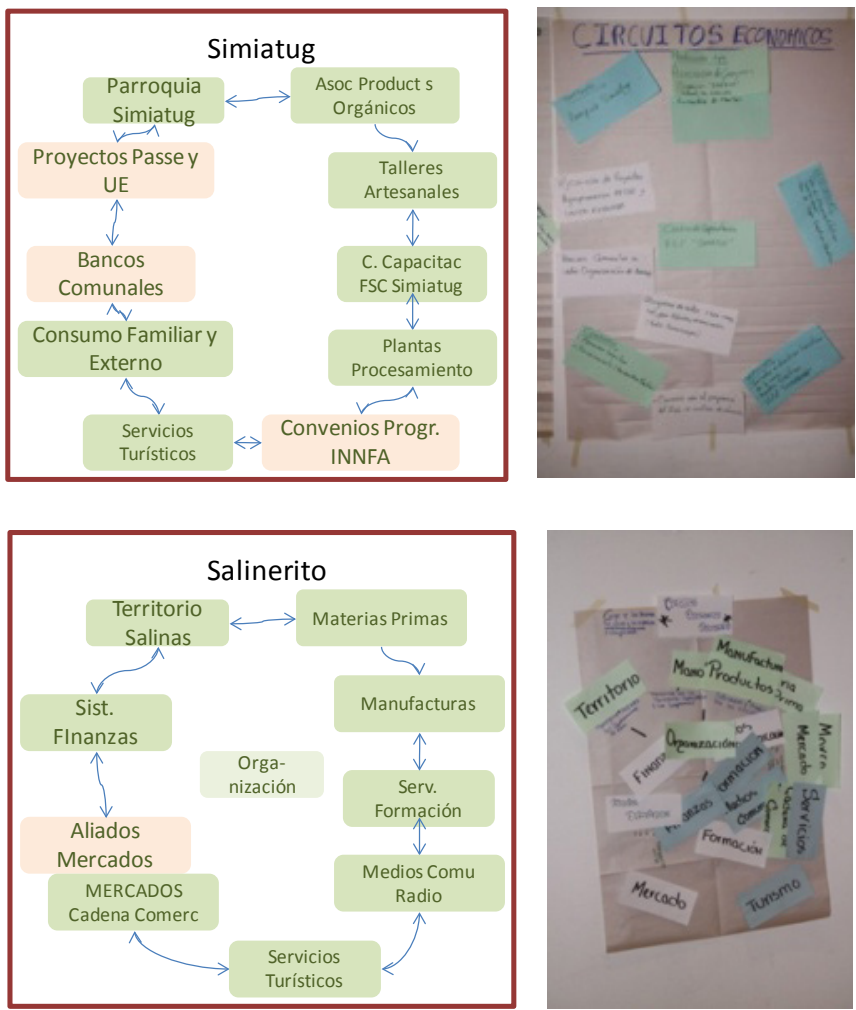

Fuente: Sistematización Proyecto Circuitos Solidarios Interculturales.

En las experiencias analizadas por el MESSE en función de los elementos de fomento de los CESI, se encontró que los circuitos habían alcanzado niveles de autonomía donde existe un mayor control del proceso económico, se habían construido redes de solidaridad, y también habían logrado construir identidad. Hay otros que se encuentran en proceso de activación, pues las alianzas y las redes son débiles y están en construcción; no obstante, los principios de solidaridad están presentes, por lo que se podría afirmar que se encuentran en transición. Existen otras experiencias de circuitos donde las prácticas son precarias y vulnerables en la construcción del proceso del circuito, los principios de la solidaridad están enunciados, pero en muchas ocasiones no se activan, no se manejan procesos, hay una baja participación en redes y dependencia de los agentes de la economía dominante. Una ampliación de ésta sistematización se puede encontrar en el libro Economía Solidaria Patrimonio Cultural de los Pueblos. (Silva, 2012)

Para el fortalecimiento de los circuitos, el MESSE ha establecido dos estrategias que permiten promover desde los territorios los CESI: los mercados solidarios y el consumo solidario. 
Mercados solidarios. Bajo la premisa de que la economía solidaria promueve sociedades con mercado y no de mercado, los mercados solidarios son espacios de coordinación de ámbitos económicos, pero también políticos, culturales, donde se generan sociabilidades importantes y las fuerzas de la oferta y demanda no determinan institucionalidades al servicio de la maximización de las ganancias. Es un espacio en disputa donde hay que ganar terreno para promover procesos de articulación promoviendo encuentros directos entre productores y consumidores, pero también de saberes, espiritualidades y festividades; en él se puede desarrollar procesos democráticos para el fortalecimiento de las prácticas solidarias y generar identidades y sentidos de pertenencia a través de normas de convivencia que no estén determinadas por la oferta y la demanda.

En este sentido es importante la apropiación de los mercados por parte de las prácticas solidarias, pero también es necesario que el estado genere políticas públicas que ayuden a promover mercados dirigidos a la economía solidaria, que estén determinados con otras normas que no sean las de la oferta y la demanda. Cabe recordar que actualmente las prácticas económicas solidarias en el país tienen una diversidad de problemas con los gobiernos locales para generar espacios de mercado. Uno de los factores es que son medidos con los mismos valores que la economía convencional, sin comprender las diferentes dinámicas que se presenta en la economía solidaria.

Consumo solidario. Otro de los elementos fundamentales para generar nuevas sensibilidades son los consumidores, partiendo de que se vive un momento donde el consumo se ha exacerbado, generando procesos "de realización humana" donde el mercado genera necesidades para el consumo superfluo. Por ello, la necesidad promover consumidores responsables, pero fundamentalmente, solidarios que se comprometan con los productores que están promoviendo formas alternativas de producir, pero además es también necesario un consumo socialmente responsable para aliviar a nuestra madre tierra. Si existe una demanda de productos elaborados por la economía solidaria se puede generar una corriente social que cambien las formas de producir, alternando la oferta, que esté al servicio de la vida, por ejemplo, en el Ecuador la campaña "que Rico es: comer Sano y de MI tierra", plantea que con 250 mil familias que demanden productos sanos se podría generar una corriente a favor de la economía solidaria. 


\section{Conclusiones}

Las prácticas económicas solidarias que se encuentran en los países andinos promueven principios de la solidaridad y la interculturalidad, las cuales están relacionadas con principios ancestrales del Don como la reciprocidad, la distribución y la complementariedad, y que se reflejan en el randi-randi, el trueque, el presta manos. Por esa razón no existe un mercado totalizado, basado en los principios de la oferta y la demanda, sino que existen formas alternativas que tienen un componente histórico cultural que están presentes, permitiendo que las otras dinámicas alternativas de hacer economía sean factibles de promover. Estas prácticas tienen un sentido altamente transformador y por lo tanto político, social, cultural y espiritual que permiten generar procesos articulados de incidencia social, ya que no son sociedades de acumulación de capital, sino que existen otras lógicas de distribución, reciprocidad y cuidado de la naturaleza.

A diferencia de las cadenas productivas, los cluster, cadenas de valor que son formas capitalistas que fomentan formas extractivitas, de explotación y de disminución de la vida, los CESI son alternativas económicas que permiten articular prácticas solidarias con el fin de que éstas rijan los procesos económicos. Los CESI son una apuesta metodológica, política, cultural y social que pretende la construcción de un sistema social y solidario, donde se fomente nuevas relaciones sociales de producción que estén marcadas por los principios de redistribución, reciprocidad y el cuidado del medio ambiente; pero también se preocupa de la construcción de fuerzas de producción donde los factores de producción, principalmente el Factor $\mathrm{C}$, y el trabajo se conviertan en categoría económicas que controlen el proceso económico.

En el país existen una diversidad de experiencias de economía solidaria presentes en la construcción de circuitos -como el fomento de los CESI- que están definidos por el control del proceso económico (producción- distribuciónconsumo- pos consumo), por el fomento de redes y alianzas que abarcan aspectos económicos, políticos, culturales y sociales, que pueden generar procesos autónomos del manejo de las prácticas sociales.

Las CESI permiten desde los territorios promover un desarrollo endógeno, que posibilita la construcción de un sistema económico y solidario a través del fomento de nuevas relaciones productivas basadas en los principios de la reciprocidad, distribución, complementariedad, vincularidad y el cuidado de 
la naturaleza. Pero, además, permite que los factores de la producción estén organizados por las categorías económicas del trabajo y la asociatividad, generando fuerzas productivas que estén al servicio de la vida. Los CESI, por su carácter articulador de fomento de alianzas puede ser implementado a nivel local, nacional e internacional, pero es en el territorio donde se deben generar procesos más sostenidos ya que están enraizados en las necesidades de las personas y la naturaleza.

Un elemento fundamental para la promover los CESI es el control del mercado. Este el espacio privilegiado para las coordinaciones a través de procesos de articulación donde las relaciones sean democráticas, participativas y fomenten el desarrollo de las capacidades; en el mercado se pueden generar procesos de construcción de identidades, valorizando los conocimientos ancestrales y sobre todo la construcción de sentido de identidad a partir de los principios solidarios. Pero además es necesario generar sensibilidades sociales con los consumidores para que estos sean responsables y solidarios, con el fin de generar comercio e intercambio justos, fomentando de esta manera relaciones más sinérgicas entre productores y consumidores.

Es necesario que el estado promueva políticas públicas que permitan aportar a la generación de los CESI, a través del mejoramiento de la calidad de los factores de producción que llegan al sector de la economía solidaria, pero además añadir valor con identidad a los productos y servicios de la economía solidaria. El estado debe promover políticas para fomentar mercados solidarios, considerando las particularidades de las prácticas económicas solidarias y facilitando espacios para la construcción de ferias, biocentros y otras formas alternativas, así como también del establecimiento de otras formas como las compras públicas y ferias inclusivas. Por otro lado, se debe promover a través del accionar del estado, consumidores solidarios a través de procesos relacionados con la educación y medios de comunicación que generen nuevas sensibilidades, nuevos sentidos comunes que fomenten la solidaridad.

Finalmente, en la medida que las prácticas solidarias se articulen a nivel local, regional e internacional con una agenda política común, podrán constituirse como un actor político y económico que genere nuevas sensibilidades, normas e instituciones, y que permita construir sociedades más solidarias que integren y fomenten la reproducción de la vida en todos los sentidos, es por eso que la economía solidaria y en particular los CESI tienen una energía transformadora que permite pensar que otro mundo es posible. 
Referencias bibliográficas

Asamblea Nacional del Ecuador. (2011). Ley de Economía Popular y Solidaria. Quito, Ecuador.

Ameglio, Pietro. (2002). Gandy y la Desobediencia Civil hoy en México. Distrito Federal, México.

Andino Verónica, (2013). RIPLES. Recuperado el 28 de Marzo de 2016, de RIPLES: http://www.reliess.org/centredoc/upload/VAndinopoltpubyecosol-Ecuador-RELIESS-final1367861067.pdf

Andrade, Cecilia; Cáceres, Milton. (2005). Cosmovisión Andina, Sumak Ally Kawsay y Economia Comunitaria. En L. G. Yolanda Jubeto, \& Guridi, Luis. (Ed.), Diálogos sobre Economia Social y Solidaria en Ecuador. Bilbao, España.

Coraggio, José Luis. (2011). Economía Social y Solidaria. Trabajo Antes que el Capital (Vol. 1). (Acosta. Alberto. Martínez, Esperanza Ed.) Quito., Ecuador: Abya Yala.

Delgado, Fredy. (2015). La Reciprocidad Andina principio de seguridad Vital. Recuperado el 30 de Marzo de 2016, de Agri Cultures: http:// www.agriculturesnetwork.org/magazines/latin-america/cultivandoecologicamente-y-comercializando-con/la-reciprocidad-andina-principio-deseguridad

Gallardo, Helio. (2001). Habitar la Tierra. Bogotá, Colombia. México D.F.: Centro de Estudios Ecuménicos, Segunda Edición, 2004.

Godelier, Mauirce. (1996). El Enigma del Don. Editorial Paidos. Buenos Aires, Argentina. 1998

Guerra, Pablo. (2005). Economia de la Solidaridad y Solidaridad en la Economía. Santiago, Chile, Univirtual.

Guridi, Luis. (2014). Fundamentos Conceptuales y Principios de la Economía Social y Solidaria. En HEGOA, (Ed.), Diálogos de la Economía Social y Solidaria en el Ecuador. Upv. Bilbao, España:

Hauwermeiren, Saar. Van. (1999). Manual de Economía Ecológica. Abya Ayala. Segunda Edición, Quito, Ecuador 
Jiménez, Jiménez. (1996). Redes Económicas de Solidaridad en Barrios Urbano Marginales de Quito. Quito, Ecuador.

Laville, Jean Louis. (2006). Definiciones Institucionales de Economía. (J.-L. Laville, Productor) Recuperado el 21 de Marzo de 2016, de Jean Louis Laville: http://www.jeanlouislaville.fr/wp-content/uploads/2009/06/Definicionese-instituciones-de-la-economia-Con-Mauss-y-Polanyi-hacia-una-Teoria-de-laEconomía-Plural.pdf

Lopera, Luz., \& Mora, Sol. (2009). ㄴos circuitos Económicos Solidarios espacios de relaciones y consensos". Semestre Económico - Universidad de Medellin, Medellín Colombia.

Mance, Euclides. (2008). La Revolución de las Redes. Varios Editores, Guanajuato, México.

Mauss, Marcel. (1971). Ensayo sobre los dones. Motivo y Forma de cambio de las sociedades primitivas. Madrid, España.

Molina, José. Luis. (2004). Manual de Antropología Económica .. Pdf. http:// abiertoelespaciolibros.blogspot.com/2009/01/jos-luis-molina-manual-deantropologa.html. Madrid, España

Max Neef, Manfred. (1993). Desarrollo a Escala Humana. Editorial NordanComunidad, Santiago, Chile.

Pereira, José. (2012). "Economía Social y Solidaria: Estudios de caso en la sierra norte del Ecuador". En MESSE, Economía Solidaria Patrimonio Cultural de los Pueblos. Editorial Abya Ayala. Quito, Ecuador.

Polanyi, Karl. (1944). La Gran Transformación. Los Origenes Politicos de Nuestros Tiempos. Fondo de Cultura Económica. Buenos Aires, Argentina:

Razeto., Luis. (2009). Economía Solidaria: Concepto, realidad y proyecto. Santiago, Chile.

(1988). Fundamentos de una Economia Comprensiva. Santia-

go,

Chile: Editores PET.

(21 de marzo de 2009). La Economía Solidaria. Concepto, realidad y Proyecto. (L. Razeto, Productor) Obtenido de LuizRazeto. net: http://www.luisrazeto.net/content/la-econom\%C3\%ADa-solidaria 
Silva, Gonzalo. (2012). Circuitos Económicos Solidarios y Puesta en Valor del patrimonio Cultural. En MESSE, \& Jiménez Jhonny. J. Vázquez (Ed.), Economía Solidaria Patrimonio Cultural de los Pueblos. Quito, Ecuador.

Wanderley, Fernanda. (2015). Desafíos Teóricos y Políticos de la Economía Social y Solidaria, Lectura desde América Latina. CIDES-UMSA. La Paz, Bolivia. Yaselga, Patricia., \& Jara, Irene. (2012). "Proceso de los Circuitos Económicos Solidarios Interculturales" Economía Solidaria Patrimonio Cultural de los Pueblos.. (J. Jiménez, Ed.) Quito, Ecuador: Abya Ayala.

Zabala, Hérnan. (1997). Circuitos Económicos Solidarios. Recuperado el 28 de marzo de 2016, de IPC: www.ipc.org.co/.../index.php?...solidaria...circuitos... solidarios...

MESSE. (2005). Movimiento de Economia Social y Solidaria, Visión. Recuperado el 30 de marzo de 2016, de MESSE: www.messe.ec 